\title{
Rapid and Non-Invasive Screening System for Early Detection of Diabetic Retinopathy via Auto Fluorescence of Falvin Proteins
}

\author{
Bin Gao ${ }^{1}$, Yang Li' ${ }^{1}$ Qifeng Jiang ${ }^{2 *}$ \\ ${ }^{1}$ Department of Medical Information, Chongqing Medical University, Chongqing, China; ${ }^{2}$ Department of Biochemistry \\ and Molecular Biology, Chongqing Medical University, Chongqing, China
}

Correspondence to: Qifeng Jiang, ‘jqf1008@aliyun.com

Keywords: Diabetic Retinopathy, Fundus Macular, Flavin Protein, Autofluorescence

Received: July 26, $2018 \quad$ Accepted: August 21, $2018 \quad$ Published: August 24, 2018

Copyright $\odot 2018$ by authors and Scientific Research Publishing Inc.

This work is licensed under the Creative Commons Attribution International License (CC BY 4.0).

http://creativecommons.org/licenses/by/4.0/

\section{(c) (i) Open Access}

\section{ABSTRACT}

Diabetic retinopathy is one of the most serious complications of diabetes, which is also one of the most important causes of blindness around the world. Autofluorescence of flavin protein in retinal pigment epithelial cells is considered as a marker of early tissue damage. This study was designed to image spontaneous fluorescence of falvin proteins in fundus macular center area. The average intensity of the fluorescence signal and the characteristics of the histogram distribution were obtained and the significant differences in the fluorescence signals between the diabetic and the normal people were also compared with statistical methods, and then a rapid and non-invasive screening method and equipment for early detection of diabetic retinopathy were developed.

\section{INTRODUCTION}

Diabetes is a metabolic disease characterized by a large amount of sugar in the blood and urine. The mechanism of diabetes is the lack of insulin secretion or insulin resistance in the patient's body. Glucose could not be fully consumed and detained in blood, causing hyperglycemia and diabetes. There are many complications caused by diabetes, including diabetic retinopathy, cardio cerebral vascular disease, macrovascular disease, diabetic nephropathy, neuropathy et al. [1]. Among them, diabetic retinopathy is one of the most serious complications of diabetes. With the increase of the incidence of diabetes, diabetic retinopathy has become the main cause of blindness in the 20 to 65 years old people, and the rate of blindness is 10 to 25 times higher than that of non-diabetic patients. It becomes more and more urgent to develop a screening system for diabetic retinopathy.

With the development of diagnostic technology at cell and molecular level, attention has been gradually attracted to autofluorescence of in situ proteins. Many studies have shown that some protein mole- 
cules in the cells have the native spontaneous fluorescence, which can be used as a "spectral biomarker" [2], and sensitive to the metabolic state of the body and the early damage of the tissues. For instance, spontaneous fluorescence of nicotinamide adenine dinucleotide (NADPH) was probed as the early carcinogenesis of epithelial cells [2], myocardial cell apoptosis [1] and early marker of tissue damage [3]. Besides, people found that long lasting hyperglycemia not only makes hemoglobin glycosylation, but also produces advanced glycation end products (AGEs). The accumulation of AGEs in skin collagen is related to the duration and severity of hyperglycemia, associating with the existence of long-term complications [4]. Based on AGEs, many screening methods for diabetic complications have been developed with skin [5] and autofluorescence of lens [6]. However, there are several disadvantages in these methods: 1) the excitation wavelength is ultraviolet (maximum excitation wavelength: $370-380 \mathrm{~nm}$ ), which is harmful to the human body with in vivo examination; 2) AGEs level was significantly influenced by age.

In diabetic patients, mitochondrial stress and apoptosis of cells caused by hyperglycemia occur shortly after the onset of the disease. This means that the measurement of mitochondrial metabolic activity can be used as early marker of disease [7]. Before apoptosis, the mitochondria in the respiratory chain show low electron transfer performance, resulting in an increase of oxidized flavin protein, which has spontaneous fluorescence properties with $460 \mathrm{~nm}$ maximum excitation and $540 \mathrm{~nm}$ maximum emission wavelength. Previous studies have shown the increase of autofluorescence might be an early marker of diabetic metabolic stress and this is especially obvious for the late mitotic cells and retinal pigment epithelial cells of the retina $[8,9]$. Because of the cellular response, early detection of retina can be performed by non-intrusive detection. Elner has confirmed this hypothesis with retinal pigment epithelial cells in vitro [10]. They found that the level of the autofluorescence of the flavin protein was well correlated with the severity of diabetic retinopathy, with nothing to do the difference of age. From this point of view, we are trying to develop a rapid and non-invasive screening method and equipment for early detection of diabetic retinopathy, based on significant differences of the autofluorescence signals of flavin protein between the diabetic and the healthy people.

\section{MATERIALS AND METHODS}

\subsection{Clinical Subjects and Sampling Group}

There was no ethical conflict with this research. All patients were informed before tests. 40 patients with diabetes mellitus (type I, 7 cases, type II, 33 cases, 20 male and 13 female) in Three Gorges Central Hospital of Wanzhou, Chongqing were selected, the average age was about 50 years old, the age range was 30 - 69 years old, according to the diabetes diagnosis standard of 1999 WHO, as the diabetic group; at the same time, there were 18 - 22 people in these two groups in terms of both eyes had no retinal complications and retinal complications in both eyes of diabetes. The negative control group had 40 normal people, with an average age of 50 years, and the age range was $30-69$ years. The blood glucose and glycosylated hemoglobin index were normal. Besides, the positive control group had 40 people, with an average age of 50 years, and the age range was 30 - 69 years, including 20 male and female, respectively. The blood glucose and glycosylated hemoglobin index were normal but with diagnosed retinopathy in positive control group.

\subsection{Cell Culture}

Human retinal pigment epithelial cells were purchased from ATCC (No. CRL-2302). All cells were maintained in DMEM:F-12 medium supplemented with $10 \%$ heat-inactivated fetal bovine serum. Cells were incubated at $37^{\circ} \mathrm{C}, 5 \% \mathrm{CO}_{2}$ and passage $3-6$ cells were used for autofluorescence measurement. Briefly, cells were incubated with different concentration of glucose for 15 days, and then the autofluorescence signals were detected in screening system at $460 \mathrm{~nm}$ maximum excitation and $540 \mathrm{~nm}$ maximum emission wavelength and the relative fluorescence intensity was obtained with ImageJ software.

\subsection{System Construction}

Based on the existing fundus camera, the basic structure of the system is retained. Only partial transforma- 
tion of the optical path system and digital transformation of the image output are carried out [Figure 1].

\subsubsection{Light Source}

As the flavin auto fluorescence needs narrowband spectroscopy, the power of the ordinary fundus camera will be significantly lower. The modified system adopts $100 \mathrm{~W} \mathrm{Hg}$ xenon lamp (Roche) as light source.

\subsubsection{Filter}

The optical path of the fundus camera is basically retained. A $460 \mathrm{~nm}$ excitation filter (bandwidth 5 $10 \mathrm{~nm}$ ) and a $540 \mathrm{~nm}$ emission filter (bandwidth $5-10 \mathrm{~nm}$ ) are added to the camera only after the light source and before the camera. These two wavelengths are the excitation peaks and emission peaks of the flavin protein autofluorescence respectively.

\subsubsection{Photomultiplier CCD (EMCCD)}

The traditional fundus camera is a film camera. In order to enable the image to be processed and displayed on the computer, the traditional film camera is changed into a digital camera. At the same time, considering the relatively weak autofluorescence of flavin protein, the highly sensitive electron multiplier CCD camera (EMCCD, Andor) is chosen.

\subsubsection{Modification}

If the imaging is blurred, the object distance in the optical path system, the distance of the image can be tuned, and an optical imaging adapter is added between the transmitting filter and the EMCCD.

\subsection{Statistical Analysis}

Data were expressed as the mean \pm SD. Statistical difference between groups was examined using the ANOVA and Student's t-test. $\mathrm{P}<0.05$ was considered as statistically significant.

\section{RESULTS}

\subsection{The Auto Fluorescence of Falvin Proteins Positively Correlated with Glucose Concentration}

To probe the relationship between the auto fluorescence of falvin proteins and glucose concentration,

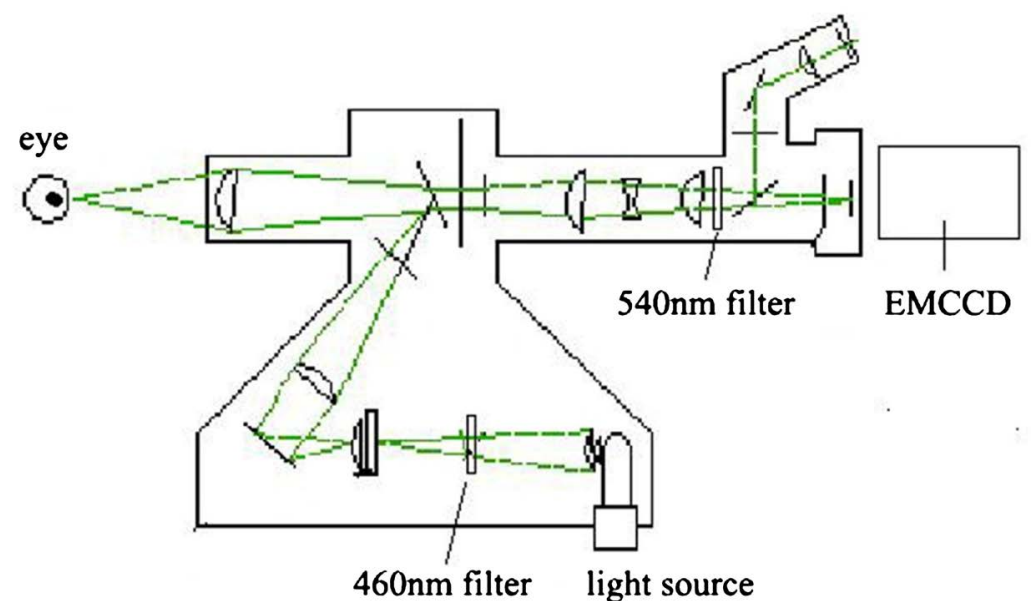

Figure 1. The basic construction of screening system for diabetic retinopathy: the light source is 100 W Hg xenon lamp; the excitation and emission filters are $460 \mathrm{~nm}$ and $540 \mathrm{~nm}$ wavelength respectively; the highly sensitive electron multiplier CCD camera is used to collected relative weak fluorescence signals of falvin proteins. 
the human retinal pigment epithelial cells were incubated with different concentration of glucose for 15 days and then the fluorescence signals from cultured cells were detected with our screening system. The relative intensity of fluorescence signals was used to demonstrate the actual effect of glucose on auto fluorescence of falvin proteins. The results showed that auto fluorescence of falvin proteins is positively correlated with glucose concentration, with the increase of glucose concentration, the auto fluorescence of cells became more and more significant and cells incubated with highest concentration of glucose presented the strongest fluorescence signals [Figure 2(a)]. The relative intensity of fluorescence also increased with the glucose concentration [Figure 2(b)].
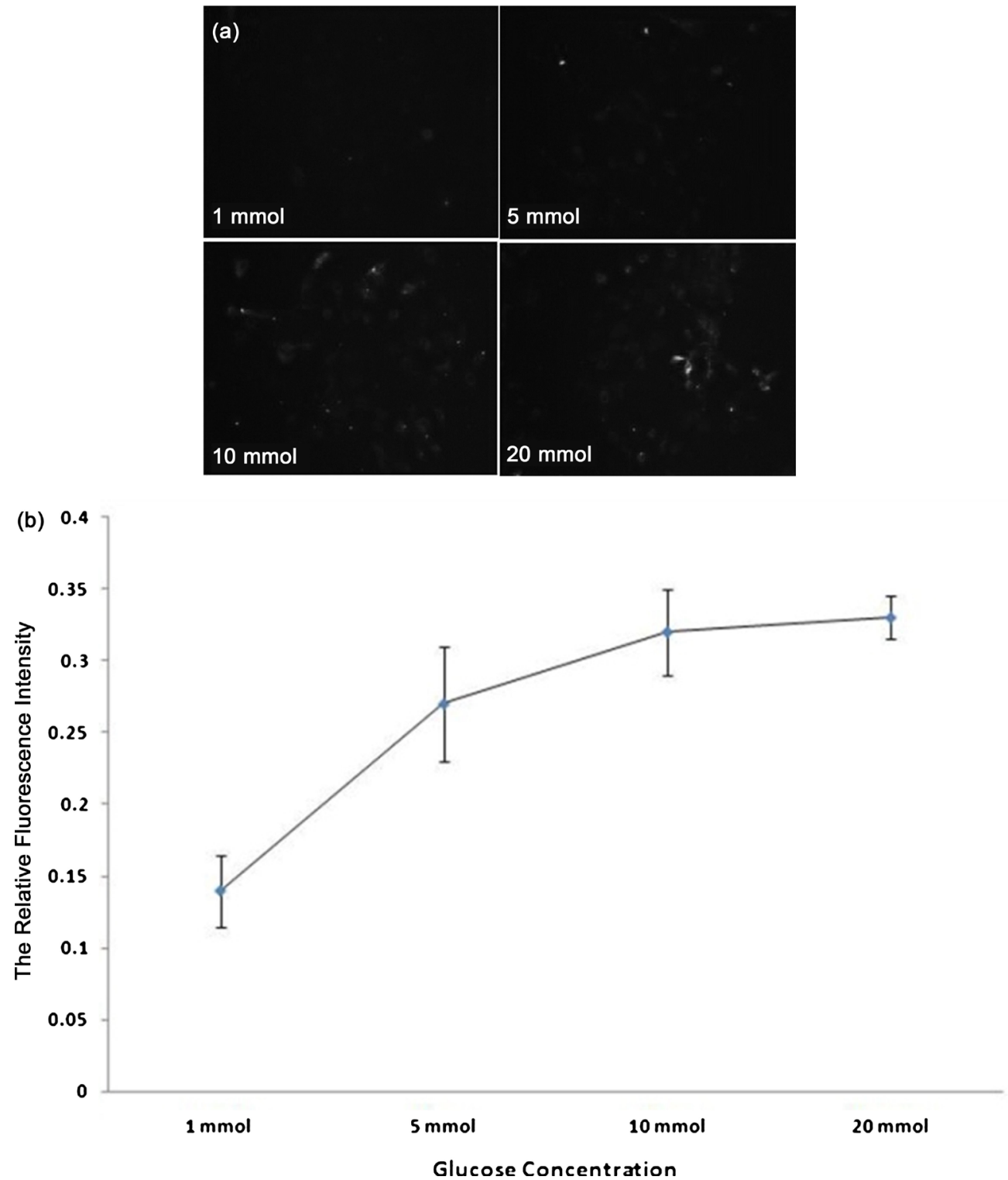

Figure 2. The fluorescence profiles of falvin proteins from human retinal pigment epithelial cells. (a) auto fluorescence of falvin proteins is positively correlated with glucose concentration. Cells presented significant fluorescence after incubation with glucose at $460 \mathrm{~nm}$ excitation and $540 \mathrm{~nm}$ emission wavelength, the fluorescence was enhanced with the increase of the glucose concentration; (b) the relative intensity of fluorescence of human retinal pigment epithelial cells in different concentration of glucose. 


\subsection{The Auto Fluorescence of Falvin Proteins Positively Correlated with Diabetic Retinopathy}

To find out the possible relationship between auto fluorescence of falvin proteins and diabetic retinopathy, 40 patients with diabetes mellitus (type I, 7 cases, type II, 33 cases) were divided into two groups, in terms no retinal complications and retinal complications in both eyes of diabetes. The negative control group had 40 healthy people with normal blood glucose and glycosylated hemoglobin index and no clinical characterization of retinopathy. Both experimental group and control group people were conducted with screening system. To specifically collect the signals of falvin proteins fluorescence, the fundus macular center area of all subjects was excited with $460 \mathrm{~nm}$ wavelength light and then the $540 \mathrm{~nm}$ wavelength fluorescence signals were promptly recorded via the highly sensitive electron multiplier CCD camera and the relative intensity of fluorescence signals was compared among control, diabetic retinopathy and diabetes without retinopathy groups. The results showed that no significant fluorescence signals could be detected in control group neither in left eyes nor in right eyes. The diabetic retinopathy group showed very strong fluorescence signals around fundus macular center areas of both left and right eyes. Besides, there were very dim signals in diabetes without retinopathy group, which barely could be detected [Figure 3(a),

(a)
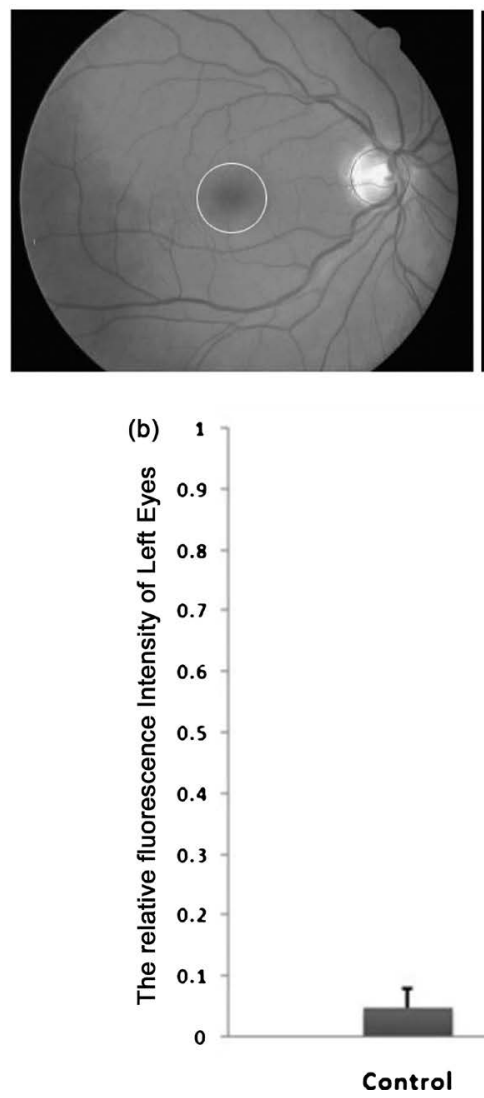

Diabetic

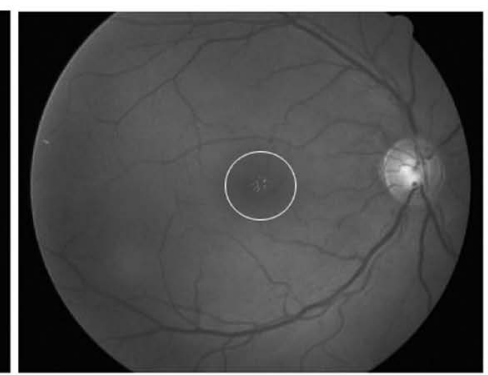

Diabetic with Retinopathy

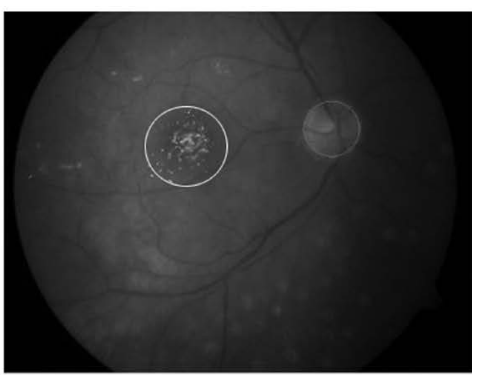

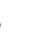


Figure 4(a)]. The relative intensity of fluorescence signals demonstrated corresponding results with the fundus fluorescence images. Diabetic retinopathy group presented significantly increase of fluorescence intensity compared with control group and diabetes without retinopathy group [Figure 3(b), Figure 4(b)].

\subsection{The Auto Fluorescence of Falvin Proteins Should Be a Specific Marker for Early Diabetic Retinopathy}

To further identify the specific role of falvin proteins auto fluorescence in diabetic retinopathy and probe the difference of fluorescence signals between diabetic retinopathy and general retinopathy, the fluorescence images of fundus macular center areas were obtained via screening system from diabetic retinopathy group and the positive control group, which included people with normal blood glucose and glycosylated hemoglobin index and diagnosed retinopathy. The fundus macular center area of all subjects was

(a)

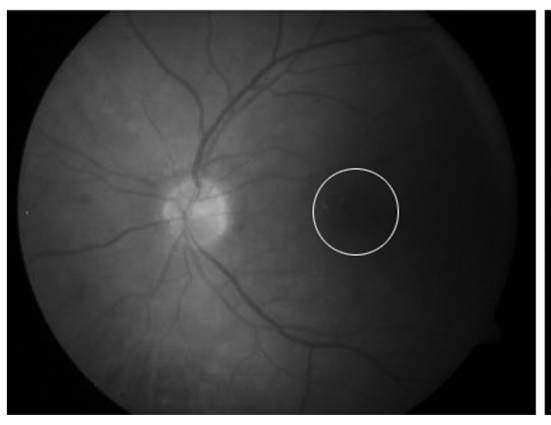

Diabetic

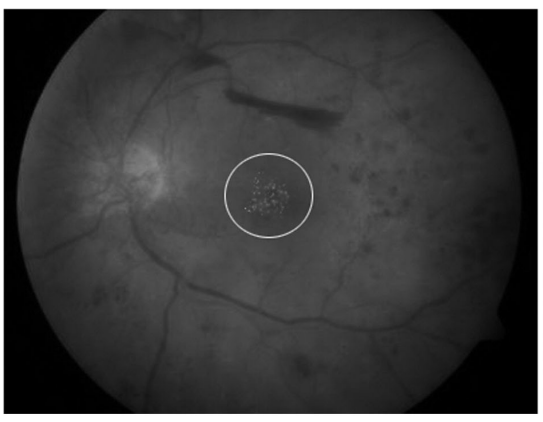

Diabetic with Retinopathy

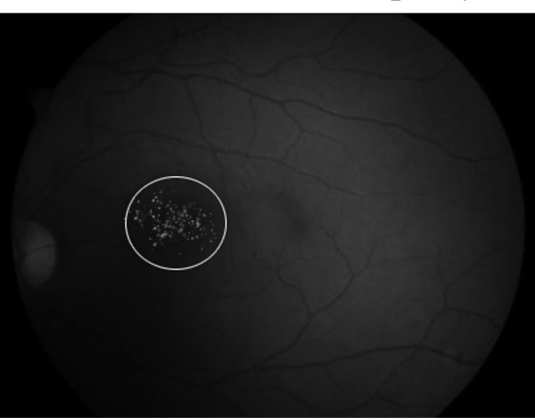

(b)

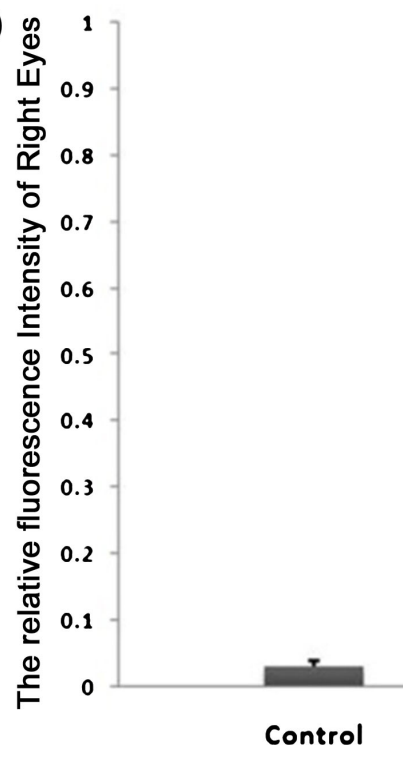

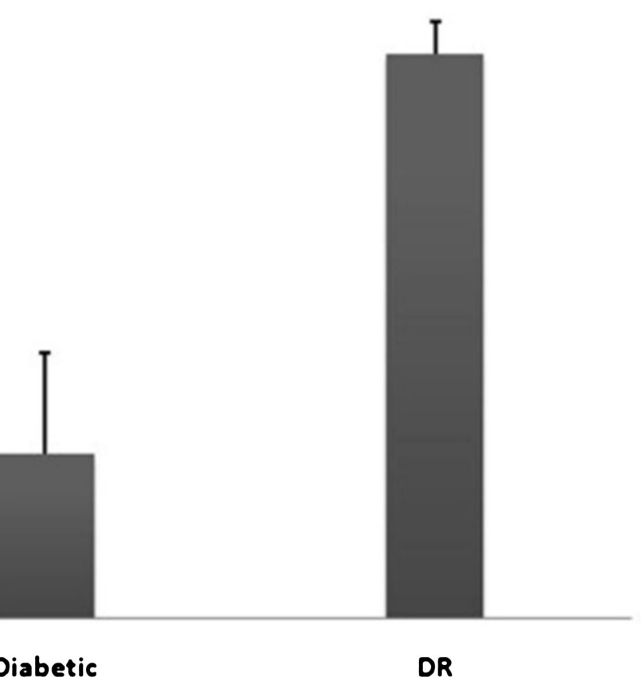

Figure 4. The fluorescence images and relative intensities of falvin proteins in fundus macular center area of right eyes. (a) the fundus macular center area (white circles) of all subjects was excited with $460 \mathrm{~nm}$ wavelength light and then the $540 \mathrm{~nm}$ wavelength fluorescence signals were promptly recorded via EMCCD. The diabetic retinopathy group showed very strong fluorescence signals compared with control group which had no significant fluorescence signals. There were very dim signals in diabetes without retinopathy group, which barely could be detected; (b) the relative intensity of fluorescence signals in each group. Diabetic retinopathy group presented significantly increase of fluorescence intensity compared with control group and diabetes without retinopathy group. 
excited with $460 \mathrm{~nm}$ wavelength light and then the $540 \mathrm{~nm}$ wavelength fluorescence signals were promptly recorded via the highly sensitive electron multiplier CCD camera and the relative intensity of fluorescence signals was compared among positive control and diabetic retinopathy group. The results showed that there were no significant fluorescence signals could be detected in fundus macular center area of positive control, with the $460 \mathrm{~nm}$ excitation and 550 emission wavelength, whereas fundus macular center area of diabetic retinopathy presented strong signals of fluorescence [Figure 5].

\section{DISCUSSION}

Diabetic patients are mainly insulin metabolic abnormalities, with dramatic changes in eye, nerve and

(a) Positive Control

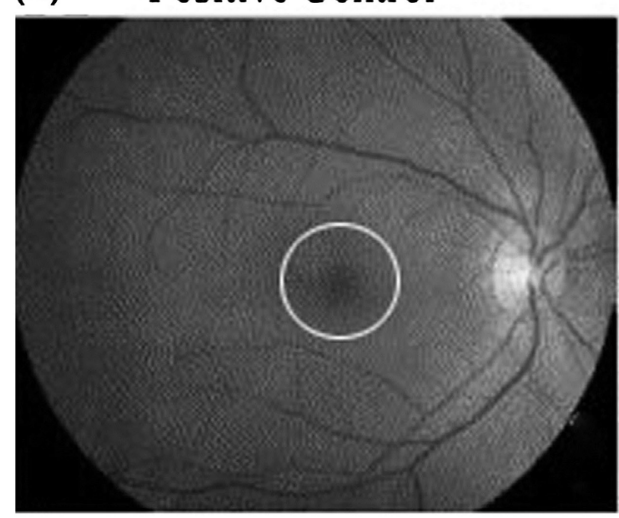

(b)$$
\text { (b) }
$$

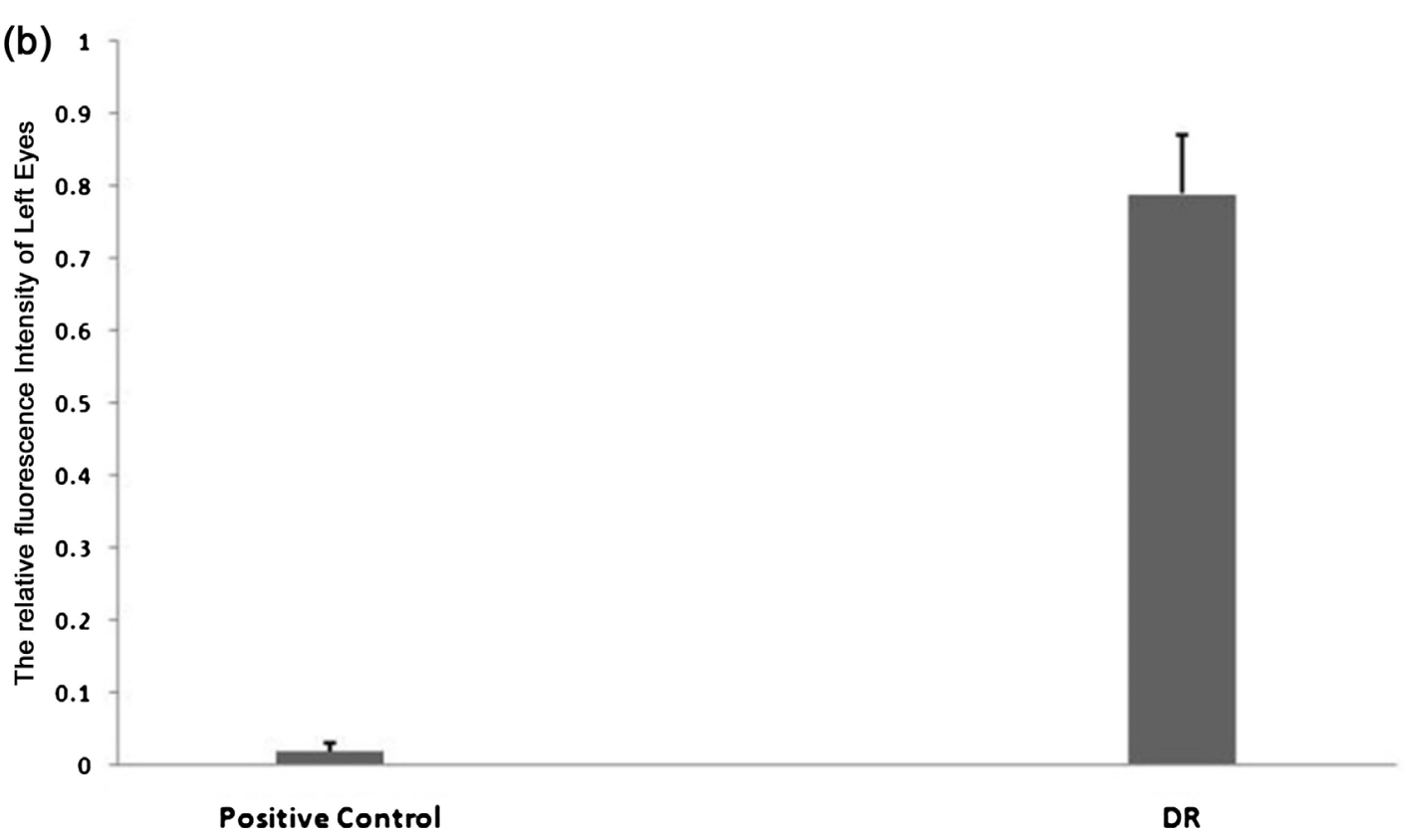

Diabetic Retinopathy

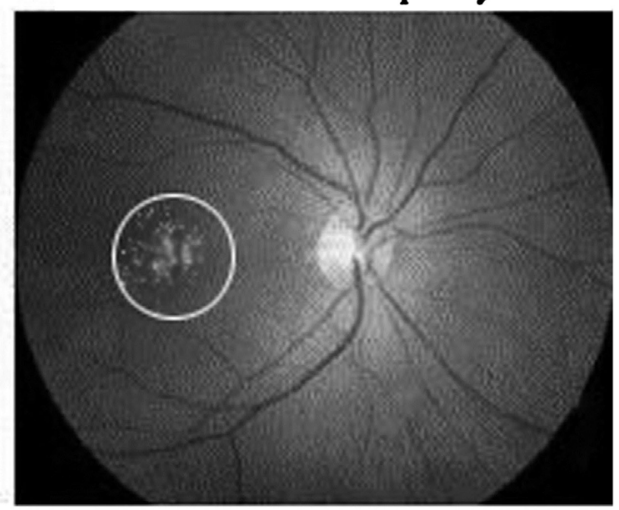

Figure 5. The comparison of fluorescence signals of falvin proteins between diabetic retinopathy and general retinopathy (positive control). (a) the fundus macular center area (white circles) of all subjects was excited with $460 \mathrm{~nm}$ wavelength light and then the $540 \mathrm{~nm}$ wavelength fluorescence signals were promptly recorded via EMCCD. The diabetic retinopathy group showed very strong fluorescence signals compared with positive control group which had no significant fluorescence signals; (b) the relative intensity of fluorescence signals in each group. Diabetic retinopathy group presented significantly increase of fluorescence intensity compared with control group. 
blood vessel microcirculation, resulting in eye nutrition and visual function damage [1]. Because of changes in blood components of diabetic patients, endothelial dysfunction is caused by impaired blood retinal barrier. The combination of retinal capillary endothelial cells and pigment epithelial cells is destroyed, resulting in the damage of the retina, which is in term of diabetic retinopathy. Diabetic retinopathy has become the main cause of blindness in the 20 to 65 years old people, and the rate of blindness is 10 to 25 times higher than that of non-diabetic patients. In this study, we are trying to develop a rapid and non-invasive screening method and equipment for early detection of diabetic retinopathy. The auto fluorescence of falvin proteins in fundus macular center area was used as marker of diabetic retinopathy and our results showed that falvin proteins fluorescence was not only positively correlated with diabetic retinopathy, but also specifically distinguished the difference between diabetic retinopathy and general retinopathy.

In the optical path, the excitation light mainly passes through the cornea, lens, vitreous body and so on. Previous studies have shown that collagen (maximum excitation wavelength: $320 \mathrm{~nm}$; maximum emission wavelength: $390 \mathrm{~nm}$ ), elastin (maximum excitation wavelength: $320 \mathrm{~nm}$; maximum emission wavelength: $400 \mathrm{~nm}$ ), AGEs (maximum excitation wavelength: $370 \mathrm{~nm}$; maximum emission wavelength: 440 $\mathrm{nm})$ are also present in the three tissues with auto fluorescence [11]. Besides, there are also several autofluorescent substances in the retinal pigment epithelium, which are lipofuscin (excitation wavelength: 450 $500 \mathrm{~nm}$; emission wavelength: $620-640 \mathrm{~nm}$ ), NADPH (maximum excitation wavelength: $340 \mathrm{~nm}$; maximum emission wavelength: $450 \mathrm{~nm}$ ), flavin protein (maximum excitation wavelength: $460 \mathrm{~nm}$; maximum emission wavelength: $540 \mathrm{~nm}$ ) [12]. So, it can be found that there are many spontaneous fluorescence signals with overlaps in the emission spectra. To reduce the interference, we use a high-tech narrow band filter (excitation filter $460 \mathrm{~nm}+5 \mathrm{~nm}$, emission filter $540 \mathrm{~nm}+5 \mathrm{~nm}$ ) to ensure that NADPH, collagen, elastin and AGEs produce only very little spontaneous fluorescence interference, because the selective excitation wavelength is longer than the excitation waves of these substances, so the spontaneous excitation of these substances is relatively weak with decrease of fluorescence. For lipofuscin, it is the main fluorescent substance of the retinal pigment epithelium and its excitation wavelength coincides with the wavelength of the flavin protein excitation, but the peak of the emission spectrum of the lipofuscin is $620-640 \mathrm{~nm}$, and the peak of the emitter of the lutein protein is about $535 \mathrm{~nm}$ [13]. By using a narrow band emission filter $(540 \mathrm{~nm}+5 \mathrm{~nm})$, the fluorescence signal on EMCCD can be ensured near the peak of the emission fluorescence of the flavin protein, but it is in a small range far away at the distal end of the lipofuscin emission fluorescence curve, so that the spontaneous fluorescence interference of lipofuscin can be reduced as much as possible. Furthermore, we chose the fundus macular center area for the autofluorescence imaging of the flavin protein. This area has two advantages: 1) the highest content of the flavin protein in the central area of the macula with the strongest signal; 2) the blood vessels in this area are much less, which would not cover the fluorescence signals of flavin protein. Besides, with less blood vessels, the interference inducing by spontaneous fluorescence of blood porphyrin derivative could be avoided [14].

In addition, the autofluorescence signal of flavin protein is very weak. In the past, because of the lack of highly sensitive imaging devices, it is difficult to collect its signal. In recent years, due to the development of optoelectronic devices, this problem has been solved. The advanced EMCCD (Electron-Multiplying CCD) technology has been able to detect single photon and achieve more than 1000 times gain amplification and our results showed that the EMCCD we chosen could not only perfectly obtain the relative weak signals of falvin proteins, but also displayed the significant differences between experimental and control group.

\section{CONCLUSION}

In this study, we are trying to develop a rapid and non-invasive screening method and equipment for early detection of diabetic retinopathy, based on significant differences of the auto fluorescence signals of flavin protein between the diabetic and the healthy people. The results showed that the auto fluorescence of flavin proteins significantly increased in fundus macular center area of patients with diabetic retinopathy, compared with healthy people or diabetes without retinopathy. Besides, auto fluorescence of flavin proteins also had significant difference between diabetic retinopathy patients and patients with general 
retinopathy, which could point out that auto fluorescence of falvin proteins should be an important marker for diabetic retinopathy.

\section{ACKNOWLEDGEMENTS}

This study was supported by NSFC (National Natural Science Foundation of China, No. 31201047 to Jiang), by SRFDP (Research Fund for the Doctoral Program of Higher Education, No. 20125503120018 to Jiang), by the open fund of Key Laboratory of Ministry of Education (No. CQKLBST-2012-005 to Jiang), by the Chongqing Yuzhong District Science and technology project (No. 20150115).

\section{CONFLICTS OF INTEREST}

The authors declare no conflicts of interest regarding the publication of this paper.

\section{REFERENCES}

1. Lotfy, M., Adeghate, J., Kalasz, H., Singh, J. and Adeghate, E. (2015) Chronic Complications of Diabetes Mellitus: A Mini Review. Current Diabetes Reviews, 13.

2. Brownlee, M. (2001) Biochemistry and Molecular Cell Biology of Diabetic Complications. Nature, 414, 813-820. https://doi.org/10.1038/414813a

3. Ceriello, A. (2006) Oxidative Stress and Diabetes-Associated Complications. Endocrine Practice, 12, 60-62. https://doi.org/10.4158/EP.12.S1.60

4. Cutler, R.G. (2005) Oxidative Stress Profiling-Part I. Its Potential Importance in the Optimization of Human Health. Annals of the New York Academy of Sciences, 1055, 93-135. https://doi.org/10.1196/annals.1323.027

5. Kowluru, R.A. and Chan, P.S. (2007) Oxidative Stress and Diabetic Retinopathy. Experimental Diabetes Research, 43603, 1-12. https://doi.org/10.1155/2007/43603

6. Desai, V., Ravi, S., Siva, P., et al. (2011) Oxidative Stress in Diabetic Retinopathy. Journal of Clinical and Diagnostic Research, 5, 994-997.

7. Sharifzadeh, M., Bernsetin, P.S. and Gellermann, W. (2006) Nonmydriatic Fluorescence-Based Quantitative Imaging of Human Macular Pigment Distribution. Journal of the Optical Society of America. A, Optics, Image Science, and Vision, 23, 2373-2387. https://doi.org/10.1364/JOSAA.23.002373

8. Du, Y., Miller, C.M. and Kern, T.S. (2003) Hyperglycemia Increases Mitochondrial Superoxide in Retina and Retinal Cells. Free Radical Biology \& Medicine, 35, 1491-1499.

https://doi.org/10.1016/j.freeradbiomed.2003.08.018

9. Ning, X., Baoyu, Q., Yuzhen, L., et al. (2004) Neuro-Optic Cell Apoptosis and Microangiopathy in KKAY Mouse Retina. International Journal of Molecular Medicine, 13, 87-92. https://doi.org/10.3892/ijmm.13.1.87

10. Elner, S.G., Elner, V.M., Field, M.G., et al. (2008) Retinal Flavoprotein Autofluorescence as a Measure of Retinal Health. Transactions of the American Ophthalmological Society, 106, 215-224.

11. Park, C.Y., Zhu, Z.J., Zhang, C., et al. (2006) Cellular Redox State Predicts in Vitro Corneal Endothelial Cell Proliferation Capacity. Experimental Eye Research, 83, 903-910. https://doi.org/10.1016/j.exer.2006.04.015

12. Tohru, A., Atsuko, A., Satoshi, I., et al. (1999) Relationship between Autofluorescence and Advanced Glycation End Products in Diabetic Lenses. Experimental Eye Research, 68, 361-366.

https://doi.org/10.1006/exer.1998.0615

13. Martin, H., Ekkehart, K. and Christiane, L. (2008) Ocular Fundus Auto-Fluorescence Observations at Different Wavelengths in Patients with Age-Related Macular Degeneration and Diabetic Retinopathy. Graefe's Archive for Clinical and Experimental Ophthalmology, 246, 105-114. 
14. Filip, S., Danijel, P.C., Naoyuki, H., et al. (2010) Monitoring Mitochondrial Electron Fluxes Using $\mathrm{NAD}(\mathrm{P}) \mathrm{H}$-Flavoprotein Fluorometry Reveals Complex Action of Isoflurane on Cardiomyocytes. Biochimica Biophysica Acta, 1797, 1749-1758. 